OPEN ACCESS

Edited by:

Ivan Zanoni,

Harvard Medical School, USA

Reviewed by:

Marco Di Gioia,

Harvard Medical School, USA

Angelo A. Manfredi,

Vita-Salute San Raffaele

University, Italy

Tracy L. McGaha,

University of Toronto, Canada

${ }^{*}$ Correspondence:

Ping-Chih Ho

ping-chih.ho@unil.ch

tThese authors have contributed equally to this work.

Specialty section:

This article was submitted to

Molecular Innate Immunity,

a section of the journal

Frontiers in Immunology

Received: 18 December 2016 Accepted: 05 April 2017

Published: 24 April 2017

Citation:

Chao T, Wang H and Ho P-C (2017) Mitochondrial Control and Guidance of Cellular Activities of T Cells.

Front. Immunol. 8:473. doi: 10.3389/fimmu.2017.00473

\section{Mitochondrial Control and Guidance of Cellular Activities of T Cells}

\author{
Tung Chao ${ }^{1,2+}$, Haiping Wang ${ }^{1,2+}$ and Ping-Chih $\mathrm{Ho}^{1,2 *}$ \\ ${ }^{1}$ Department of Fundamental Oncology, University of Lausanne, Lausanne, Switzerland, ${ }^{2}$ Ludwig Center for Cancer \\ Research, University of Lausanne, Lausanne, Switzerland
}

Immune cells protect us against infection and cancer cells, as well as functioning during healing processes to support tissue repairing and regeneration. These behaviors require that upon stimulation from immune activation the appropriate subsets of immune cells are generated. In addition to activation-induced signaling cascades, metabolic reprogramming (profound changes in metabolic pathways) also provides a novel form of regulation to control the formation of desirable immune responses. Immune cells encounter various nutrient compositions by circulating in bloodstream and infiltrating into peripheral tissues; therefore, proper engagement of metabolic pathways is critical to fulfill the metabolic demands of immune cells. Metabolic pathways are tightly regulated mainly via mitochondrial dynamics and the activities of the tricarboxylic acid cycle and the electron transport chain. In this review, we will discuss how metabolic reprogramming influences activation, effector functions, and lineage polarization in T cells, with a particular focus on mitochondria-regulated metabolic checkpoints. Additionally, we will further explore how in various diseases deregulation and manipulation of mitochondrial regulation can occur and be exploited. Furthermore, we will discuss how this knowledge can facilitate the design of immunotherapies.

Keywords: immunometabolism, mitochondrion, macrophage, T cell, oxidative metabolism

\section{INTRODUCTION}

Immune cells circulate throughout our bodies and reside in peripheral tissues where they perform many vital functions, including providing protection against pathogens, impeding cancer cells, and supporting tissue homeostasis. How immune cells respond to the nutrient state of various tissues and whether their nutrient-sensing mechanisms regulate immune responses are fascinating questions yet to be resolved. Research accumulated in the past decade has revealed that immune cells change their metabolic programs to support activation and differentiation $(1,2)$. Moreover, protective immune responses may be detrimentally affected by metabolic stress caused by nutrient deprivation, coinhibitory receptors, and production of certain metabolites. By contrast, targeting the metabolic machineries that immune cells exploit during activation can lead to promising outcomes with improved antitumor immunity and autoimmunity in preclinical animal models $(3,4)$. These findings expose the importance of metabolic reprogramming in guiding immune responses and suggest that the nutrient composition of where the immune cell resides and infiltrates may be a critical regulator in orchestrating immune responses.

Mitochondrion is the metabolic hub of the cell that governs energy production through coordination of the electron transport chain (ETC) and the tricarboxylic acid (TCA) cycle. In addition to 
energy production, mitochondrion catabolizes nutrients, including glucose, amino acids, and fatty acids, to produce building blocks for cell growth and expansion (5). In order to meet their metabolic demands, cells have to change their mitochondrial dynamics, including mitochondrial volume, structure, membrane potential $(\Delta \Psi \mathrm{m})$, and location, in response to nutrient availability and growth stimuli. Although research on how mitochondrial dynamics impact cellular metabolism have been mainly conducted in cancer and stem cells (6-8), several recent studies have revealed that this process may play critical roles in immunometabolic regulation in both innate and adaptive immune cells (9). In this review, we will summarize how T cells control mitochondrial dynamics in order to meet their metabolic demands. Furthermore, we will discuss how mitochondria-derived signals potentially guide $\mathrm{T}$ cell activation and differentiation.

\section{MITOCHONDRION: THE CENTRAL REGULATOR OF CELLULAR METABOLISM AND BEYOND}

\section{Mitochondria Are the Power Plant for Cellular Bioenergetic Demands}

Mitochondria are the major energy producers within a cell that couple metabolite oxidation to aerobic respiration (10). Glucose and fatty acids, after being catabolized through glycolysis and $\beta$-oxidation, respectively, fuel the TCA cycle in the form of acetyl-CoA. Acetyl-CoA is further oxidized into carbon dioxide to generate $\mathrm{NADH}$ and $\mathrm{FADH}_{2}$, the main sources of electrons for the ETC. The ETC ultimately transfers electrons provided by $\mathrm{NADH}$ and $\mathrm{FADH}_{2}$ to oxygen, while concurrently generating $\Delta \Psi \mathrm{m}$ across the mitochondrial inner membrane. This proton gradient is further utilized to produce ATP (9). In addition to generating ATP, mitochondria also contribute to lipid and amino acid synthesis to build macromolecules. Acetyl-CoA can be transported out of the mitochondria into the cytosol where it is used for protein acetylation as well as de novo fatty acid synthesis (11). Citrate can also be exported into the cytosol for de novo fatty acid synthesis and into the nucleus to serve as an acetyl-CoA donor for histone modification (12). Furthermore, the metabolic intermediates of the TCA cycle can also be used for production of cholesterol, nucleotides, and amino acids. These processes provide building blocks for cell proliferation and growth at a cost of TCA cycle metabolite depletion. Altogether, mitochondria bridge nutrient metabolism to fulfill the bioenergetic demands of cell through the coordination of TCA cycle and ETC.

\section{Mitochondrial Dynamics under Metabolic Stress and Reprogramming}

Mitochondrial quality and activities are maintained and adjusted through mitochondrial dynamics. In response to the type, strength, and duration of metabolic or genomic stress, mitochondrial dynamics regulate mitochondrial fusion, fission, mobility, biogenesis, and degradation. Mitochondrial mobility regulates the subcellular localization of mitochondria, whereas mitochondrial fusion and fission controls mitochondria mass and metabolism by fusing (fusion) or splitting (fission) the inner and outer membranes and matrix components $(13,14)$. Nutrient deprivation induces the formation of a tubular network of mitochondria by promoting mitochondrial fusion and suppressing mitophagy (a mitochondrial clearance process) (15). By contrast, severe and prolonged DNA damage leads to mitochondrial fission and further facilitates the clearance of damaged mitochondria via mitophagy (16). Thus, mitochondrial fusion and fission provide a method to efficiently and flexibly adjust mitochondrial quality and quantity. Importantly, most of the mitochondrial fusion and fission machineries are conserved from yeast to mammals, further implicating the importance of these processes (17). Mechanistically, mitofusin and optic atrophy 1 (OPA1) are two dynamin-like GTPases that control fusion of the mitochondrial outer and inner membranes, respectively (18). These proteins are regulated by ubiquitination and proteolytic cleavage. When mitophagy is induced, mitofusin 1 and 2 are ubiquitinated in a PTEN-induced putative kinase 1/Parkin-mediated manner (19). Moreover, OPA 1 is constitutively cleaved by protease Yme $1 \mathrm{~L}$ in the intermembrane space in order to shape proper cristae structures (20). Furthermore, loss of mitochondrial $\Delta \Psi \mathrm{m}$ induces OPA1 cleavage by protease OMA1, a process that further dampens mitochondrial fusion $(21,22)$. Conversely, mitochondrial fission is triggered by phosphorylation of dynamin-related protein 1 (Drp1) on serine 616 by protein kinase C. This event promotes Drp1 translocation to the mitochondrial outer membrane and facilitates the association between Drp1 with other adaptor proteins, including Fis1 (mitochondrial fission 1 protein), Mff (mitochondrial fission factor), and MiD49/51 (23-25). By contrast, phosphorylation of Drp1 on serine 637 by protein kinase A (PKA) leads to Drp1 inactivation (26). Additionally, mitofusin 2 is regulated via JNK phosphorylation, which when coordinated with Huwe1-regulated ubiquitination promotes stress-induced mitochondrial fragmentation and apoptotic cell death (27). These regulatory steps tightly control the balance of mitochondrial fusion and fission to actively fine-tune the mitochondria's metabolic activity. Mitochondrial fusion can increase cristae formation and respiratory complex formation as well as increasing the substrate uptake fueling oxidative phosphorylation (OXPHOS). Moreover, fusion also promotes fatty acid oxidation (FAO), which is important for the formation and survival of memory $\mathrm{T}$ cells (discussed in Section "Metabolic Reprogramming Fuels T Cell Activation and Differentiation") (28). On the other hand, mitochondrial fission not only acts to eliminate dysfunctional mitochondria but is also an adaptation that occurs in response to increased aerobic glycolysis (29).

\section{METABOLIC REPROGRAMMING FUELS T CELL ACTIVATION AND DIFFERENTIATION}

During viral infection, T cell activation occurs via several distinct phases. Cell growth is the initial phase, with subsequent massive clonal expansion and differentiation, followed by an abrupt contraction phase, and then persistent long-lived memory $\mathrm{T}$ cells (30). During the initial growth phase, $\mathrm{T}$ cells undergo an activation-induced reprogramming of their metabolism. FAO in naive $\mathrm{T}$ cells is converted to anabolic metabolism in activated 
T cells, including increased aerobic glycolysis, pentose-phosphate pathway, and glutaminolysis $(31,32)$. Mitochondrial biogenesis and proteomic reprogramming are induced upon $\mathrm{T}$ cell receptor (TCR) activation in T cells. Mitochondrial regulations strongly activate one-carbon metabolism fueled by serine and glycine metabolism, which is essential for redox homeostasis as well as purine and thymidine formation $(33,34)$. In contrast to cytotoxic effector $\mathrm{T}$ cells, memory $\mathrm{T}$ cells possess elevated mitochondrial mass and use fatty acids as the main energy source through FAO $(35,36)$. Interestingly, helper effector T cells (Th1, Th2, or Th17) use aerobic glycolysis to support their effector functions and differentiation programs similar to cytotoxic $\mathrm{CD}^{+} \mathrm{T}$ cells, whereas regulatory $\mathrm{T}$ cells utilize FAO to maintain lineage stability and immunosuppressive functions (37-39). Several recent reviews have summarized how these metabolic pathways intertwine with $\mathrm{T}$ cell activation and differentiation $(3,40)$. Therefore, we will focus on how mitochondria-derived regulations are involved in these processes.

\section{Mitochondria-Governed Metabolic Regulations in Memory T Cells}

Unlike the short-lived effector T cells that rely on aerobic glycolysis, memory $\mathrm{T}$ cells display increased mitochondrial mass, spare respiratory capacity, and utilize FAO to meet their metabolic demands $(35,41)$. Reduced glucose uptake and glycolysis has been shown to not only suppress effector $\mathrm{T}$ cell functions but also promote $\mathrm{T}$ cell differentiation toward memory-like precursor cells that have an elevated dependence on mitochondrial metabolism (42). Additionally, it has been shown that IL-15, an important cytokine for the survival of memory $\mathrm{T}$ cells, increases mitochondrial mass and copy numbers in memory T cells $(35,43)$. Furthermore, the elevated oxidative metabolism and mitochondrial activity in memory $T$ cells could positively impact survival and the rapid transition to glycolysis after re-stimulation $(36,41$, 44). These findings suggest that mitochondrial mass and activity play critical roles in guiding the generation of memory $\mathrm{T}$ cells. Interestingly, mitochondrial dynamics have also been reported to modulate the differentiation process in effector and memory $\mathrm{T}$ cells. Effector T cells primarily possess fragmented mitochondria, which might facilitate aerobic glycolysis. During TCR activation by antigen-presenting cells, Drp1-mediated mitochondrial fission and translocation of mitochondria to the immune synapse could be promoted by suppression of PKA-induced Drp 1 phosphorylation at serine 637 by mTOR activation and the calcium $\left(\mathrm{Ca}^{2+}\right)$ flux-mediated phosphatase calcineurin (45-48). By contrast, Opa1-dependent mitochondrial fusion promotes memory $\mathrm{T}$ cell formation and oxidative metabolism by maintaining the tightly associated cristae structures and ETC complexes (Figure 1). Notably, OXPHOS can stimulate mitochondrial inner membrane fusion through Yme1L-mediated cleavage of Opa1 (49). In addition, oxidative stress-induced glutathione oxidation results in elevated mitochondrial fusion by promoting mitofusin dimerization (50). Moreover, mitochondrial deacetylase sirtuin-3 (SIRT3) deacetylates OPA1, which activates OPA1's GTPase activity promoting mitochondrial networking and preventing cell death (51). Thus, TCR signaling and metabolites derived from oxidative metabolism contribute to the regulation of $\mathrm{T}$ cell mitochondrial dynamics.

In addition to regulating mitochondrial structure, metabolites derived from mitochondrial metabolic pathways may also regulate $\mathrm{T}$ cell differentiation by affecting the epigenetic and transcriptomic landscape. One recent study demonstrated that S-2-hydroxyglutarate (S-2HG) can act as an immunometabolite altering the gene expression profile with changes in the epigenetic landscape toward memory-like CD8 ${ }^{+} \mathrm{T}$ cells (52). Since S-2HG is a chemical analog of $\alpha$-ketoglutarate and succinate, we postulate that production of $\alpha$-ketoglutarate and succinate could regulate $\mathrm{T}$ cell differentiation by modulating Jumonji D3 (Jmjd3)- and ten eleven translocation (Tet)-dependent histone and DNA methylation, respectively $(53,54)$. These mitochondria-derived metabolites may provide signals that impact differentiation, exhaustion, and the secretome of T cells through mitochondrianucleus communication. Thus, future investigations to determine if mitochondrial dynamics and activity can modulate $\mathrm{T}$ cell differentiation through as yet undefined mitochondria-nucleus communication will provide exciting new insights for immunometabolic regulation.

\section{T Cell Exhaustion during Viral Infection and in the Tumor Microenvironment}

$\mathrm{T}$ cell exhaustion describes a state when $\mathrm{T}$ cells are incapable of proliferating or producing effector molecules, which often occurs as a result of chronic antigen exposure in diseases such as viral infections and tumors (55). Although changes in the transcription program of $\mathrm{T}$ cells can contribute to $\mathrm{T}$ cell exhaustion, recent studies have revealed that metabolic insufficiency and deregulation of nutrient-sensing pathways also contribute to $\mathrm{T}$ cell exhaustion (56). During chronic lymphocytic choriomeningitis virus infection, Bengsch et al. reported that glycolytic and mitochondrial metabolism in early effector $\mathrm{CD} 8^{+} \mathrm{T}$ cells are repressed by programmed cell death protein 1 (PD-1) signaling. PD-1 signals also suppress the expression of peroxisome proliferatoractivated receptor gamma coactivator 1-alpha (PGC1 $\alpha$ ) that inhibits mitochondrial biogenesis in viral-specific $\mathrm{CD}^{+} \mathrm{T}$ cells leading to declined effector function in exhausted $\mathrm{T}$ cells (57). Intriguingly, PGC1 $\alpha$ overexpression in exhausted $\mathrm{T}$ cells is able to enhance their effector functions and improve mitochondrial biogenesis. By contrast, it has been reported that HIV-specific $\mathrm{CD}^{+} \mathrm{T}$ cells display increased mitochondrial mass, resulting in higher cluster of differentiation 95 (CD95)/CD95-ligand induced apoptosis $(28,58,59)$. Thus, it is important to further investigate the contributions of mitochondrial biogenesis on $\mathrm{T}$ cell exhaustion and how we can target mitochondrial metabolism of T cells when treating chronic viral infection.

The tumor microenvironment can also cause metabolic insufficiency in T cells by reducing PGC1 $\alpha$-mediated mitochondrial biogenesis (60). Similar to chronic viral infection, overexpressing PGC1 $\alpha$ in tumor antigen-specific $\mathrm{CD}^{+} \mathrm{T}$ cells sustains their metabolic fitness and improves their antitumor responses in the tumor microenvironment. Of note, PD-1 signal is dispensable in tumor-induced impairment of mitochondrial biogenesis in $\mathrm{T}$ cells. Since hypoxia and nutrient deprivation caused by 


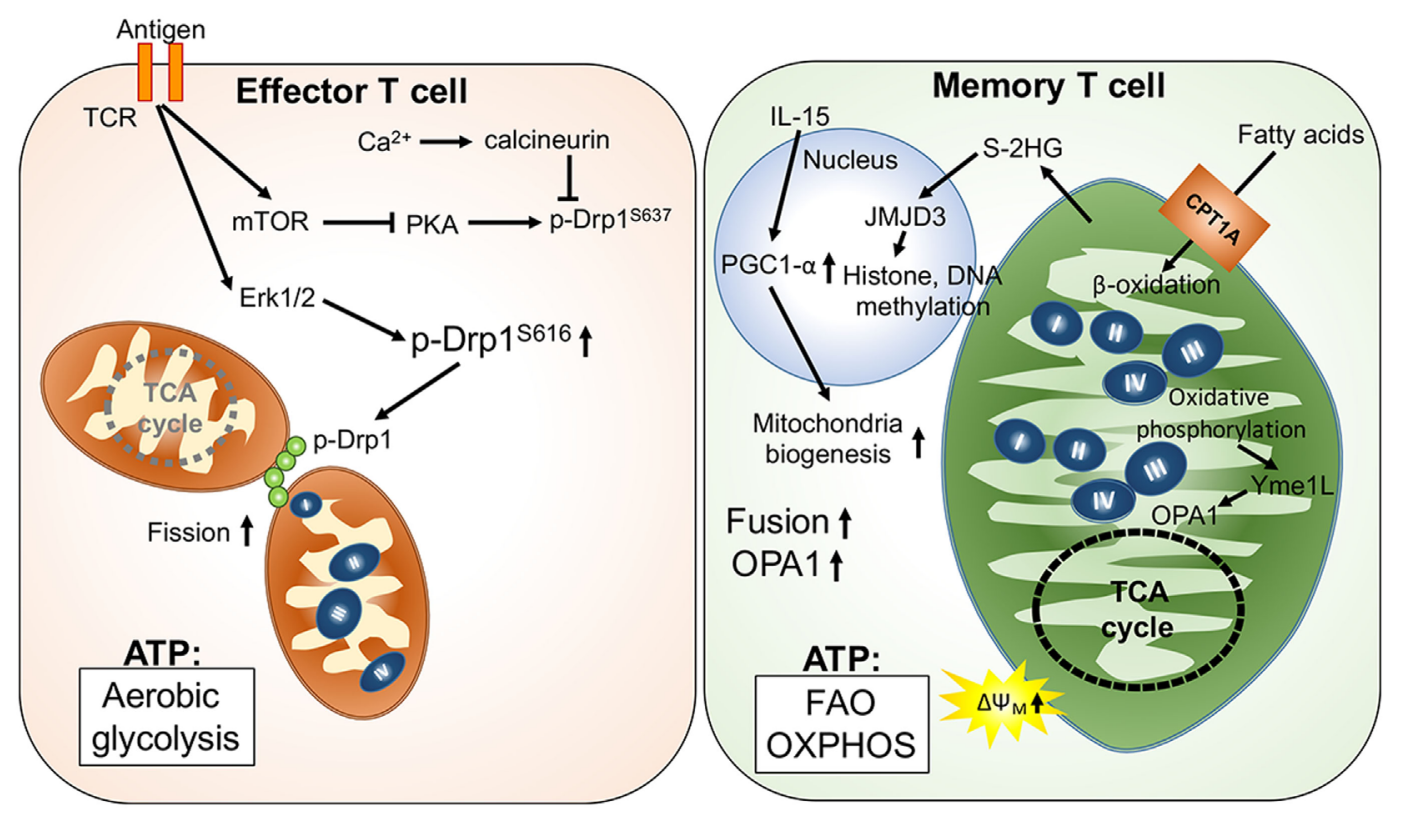

FIGURE 1 | Mitochondria-governed metabolic regulations upon T cell activation and memory T cell formation. T cell receptor (TCR) activation signaling facilitates mitochondrial fission, leading to looser cristae structures, and lower respiratory functions. In this scenario, ATP production is mainly from aerobic glycolysis. By contrast, fusion of mitochondria is facilitated by upregulation of optic atrophy 1 (OPA1)-mediated mitochondrial inner membrane fusion in memory $\mathrm{T}$ cells. Furthermore, the tight cristae structure and compact respiratory complex also support maintenance of higher mitochondrial membrane potential $(\Delta \Psi \mathrm{m})$ and the oxidative metabolism in mitochondria.

elevated tumor metabolism could drastically affect mitochondrial metabolism and dynamics (61), it is likely that T cells fail to sustain their mitochondrial activity and biogenesis due to a metabolic status in the tumor microenvironment that does not exist in the chronic viral infection condition. Interestingly, PD-1 signaling has been shown to increase FAO and mitochondrial spare respiratory capacity in effector T cells (62). Therefore, we postulate that the impairment in mitochondrial biogenesis while receiving PD-1 signaling might result in cell death and senescence in tumor-infiltrating $\mathrm{T}$ cell due to metabolic catastrophe (Figure 2). Altogether, these recent studies in chronic viral infection and tumors suggest that mitochondrial metabolism in $\mathrm{T}$ cells could critically regulate $\mathrm{T}$ cell immune responses in disease progression. Further investigations are needed to reveal how mitochondrial activity is connected with $\mathrm{T}$ cell immune responses and whether regulation of mitochondrial dynamics also controls $\mathrm{T}$ cells behavior. These investigations will provide novel insights for immunometabolic regulation and facilitate the design of immunotherapies.

\section{T Cell Aging}

Accumulation of dysfunctional mitochondria is frequently observed in aged cells (63). The mitochondrial free radical theory of aging has been recognized for decades (64); oxidation of cellular macromolecules and mitochondrial DNA damages are thought to be mainly caused by reactive oxygen species (ROS) produced from dysfunctional mitochondria $(65,66)$. Aged T cells display a reduction in respiratory metabolism and ETC activity; this is accompanied by reduced $\mathrm{Ca}^{2+}$ influx into mitochondria and elevated mitochondrial ROS production. These changes could lead to decreased $\Delta \Psi \mathrm{m}$ and ATP production, as well as activation of nuclear factor of activated T-cells (67). Aged T cells may also upregulate the expression levels of coinhibitory receptors such as PD-1, TIGIT (T-cell immunoreceptor with Ig and ITIM domains), Lag-3 (lymphocyte-activation protein 3), and Tim-3 (T-cell immunoglobulin and mucin-domain containing-3). Therefore, two attractive strategies to rejuvenate the immune responses of aged $\mathrm{T}$ cells are (1) activate mitochondrial biogenesis and (2) improve mitophagy-mediated mitochondrial quality control. Among the proposed strategies, nicotinamide adenosine dinucleotide (NAD)-dependent regulation has delivered promising outcomes in different aging studies (68). Manipulating cellular metabolism or treating with nicotinamide riboside increases NAD and may prevent aging by restoring mitochondrial activity. The restoration of mitochondrial activity occurs mainly because of mitochondrial biogenesis activation induced by a sirtuin 1-PGC1 $\alpha$ pathway and SIRT3-induced mitophagy $(69,70)$. However, it remains unclear if these strategies can also be applied to aged T cells. Detailed analysis of the contributions of deregulated mitochondrial dynamics in $\mathrm{T}$ cell aging will provide a springboard for developing immunometabolic boosters to rejuvenate aged $\mathrm{T}$ cells.

\section{T Cells in Autoimmunity}

A well-functioning immune system is reliant on the selection of a lymphocyte repertoire that is adequately diverse in response to numerous foreign antigens yet sufficiently self-tolerant to avoid the development of autoimmunity. As described above, 


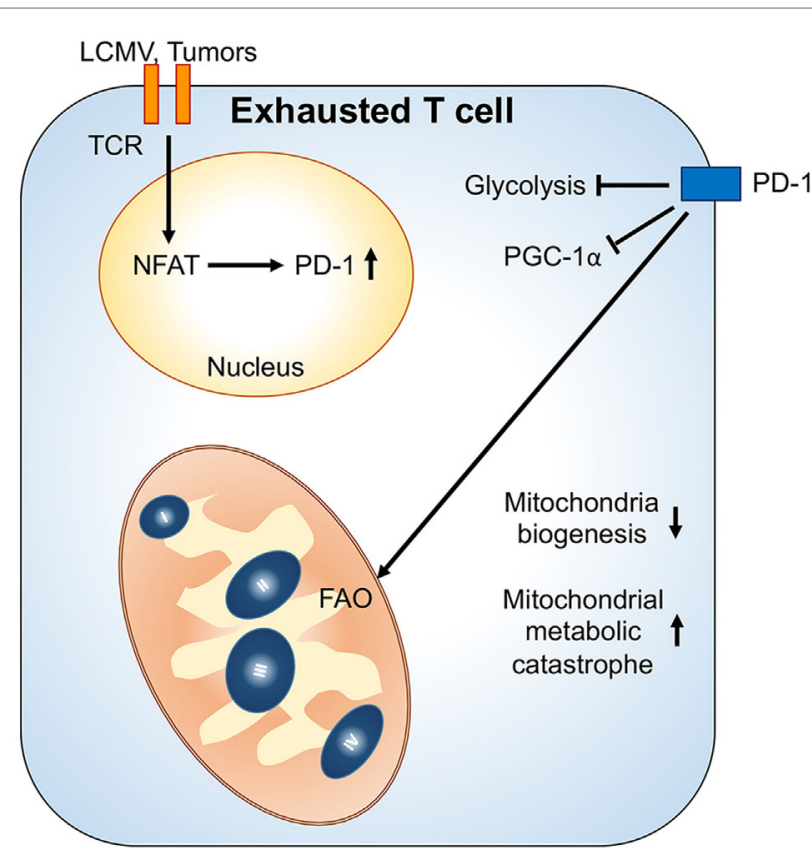

FIGURE 2 | Mitochondrial metabolic reprogramming under exhaustion of T cells. Upon chronic antigenic stresses such as lymphocytic choriomeningitis virus (LCMV) clone 13 infection and tumors, sustained T cell receptor (TCR) activation leads to NFAT-mediated programmed cell death protein 1 (PD-1) upregulation. Upregulation of PD-1 and chronic TCR activation suppress PGC1 $\alpha$ expression, leading to decrease of mitochondria biogenesis. Moreover, PD-1 signal would suppress glycolysis and promote mitochondrial metabolism toward fatty acid oxidation (FAO). These metabolic reprogramming in mitochondria may further cause mitochondrial metabolic catastrophe if there is overload of mitochondrial oxidative metabolisms without sufficient antioxidant responses.

production of ROS and ATP synthesis, which are tightly associated with the $\Delta \Psi \mathrm{m}$, regulate the activation, proliferation, and selection of the cell death pathway in T cells. Recent evidence has proposed that the regulation of programmed cell death in $\mathrm{T}$ cells is impaired in both human and murine systemic lupus erythematosus (SLE) contributing to other disease pathogenesis (71). Meanwhile, $\Delta \Psi \mathrm{m}$ and ROS levels are elevated in patients with SLE in comparison with healthy controls (72). When mitochondrial ROS was blocked in lupus T cells, mice showed reduced interferon pathway signaling and had decreased signs of autoimmune disease (73). Furthermore, coordinated mitochondrial hyperpolarization and ATP depletion play key roles in abnormal T cell death in lupus patients (74). Hence, persistent mitochondrial hyperpolarization, which leads to increased ROS

\section{REFERENCES}

1. Pearce EL, Pearce EJ. Metabolic pathways in immune cell activation and quiescence. Immunity (2013) 38(4):633-43. doi:10.1016/j.immuni.2013. 04.005

2. O'Neill LA, Kishton RJ, Rathmell J. A guide to immunometabolism for immunologists. Nat Rev Immunol (2016) 16(9):553-65. doi:10.1038/nri. 2016.70

3. O'Sullivan D, Pearce EL. Targeting T cell metabolism for therapy. Trends Immunol (2015) 36(2):71-80. doi:10.1016/j.it.2014.12.004 production and ATP depletion, might be responsible for the unusual increase in spontaneous apoptosis in SLE patients and other relative autoimmune diseases. However, the relative impact and metabolic signaling pathways involved still require further investigation. This will likely allow us to identify novel targets for pharmacological intervention in patients with autoimmune diseases.

\section{CONCLUSION REMARKS}

Over the past two decades, studies have revealed that metabolic checkpoints couple metabolic demands and activation signaling cascades to orchestrate immune cell differentiation, proliferation, survival, and function, through multiple layers of regulation. These regulations are operated through the most ancient cellular machineries to ensure proper immune responses are engaged. In this review, we discuss how mitochondrial dynamics can support T cell metabolic demands and the contribution of mitochondrial dynamics to $T$ cell behavior in different diseases. Nevertheless, the detailed regulation and contribution of mitochondrial dynamics to immune cells remains to be fully explored. Most importantly, deciphering how mitochondrial activity communicates with the nuclear landscape, including changes in epigenetics and the transcriptome, will provide novel information regarding immunometabolic regulation and cell biology. We also envisage that these mitochondria-derived regulations may be utilized in other immune cells, including antigen-presenting cells, NK cells, and innate lymphocytes. Altogether, understanding these underlying immunometabolic controls will provide the foundations for novel immunotherapies that can selectively tune the immune responses of deregulated immune cells in various diseases, including autoimmunity and cancer.

\section{AUTHOR CONTRIBUTIONS}

All authors listed have made substantial, direct, and intellectual contribution to the work and approved it for publication.

\section{FUNDING}

$\mathrm{P}-\mathrm{CH}$ is supported in part by Swiss Institute for Experimental Cancer Research (26075483), SNSF project grant (31003A 163204), Harry J. Lloyd Charitable Foundation, Roche pRED grant, Swiss Cancer League grant (KFS-3949-08-2016), and Anna Fuller Fund. P-CH is a recipient of Melanoma Research Alliance Young Investigator Award. TC is supported by University of Lausanne FBM PhD fellowship.

4. Yin Y, Choi SC, Xu Z, Perry DJ, Seay H, Croker BP, et al. Normalization of CD4+ T cell metabolism reverses lupus. Sci Transl Med (2015) 7(274):274ra18. doi:10.1126/scitranslmed.aaa0835

5. Ahn CS, Metallo CM. Mitochondria as biosynthetic factories for cancer proliferation. Cancer Metab (2015) 3(1):1. doi:10.1186/s40170-0150128-2

6. Todd LR, Damin MN, Gomathinayagam R, Horn SR, Means AR, Sankar U. Growth factor erv1-like modulates Drp1 to preserve mitochondrial dynamics and function in mouse embryonic stem cells. Mol Biol Cell (2010) 21(7):1225-36. doi:10.1091/mbc.E09-11-0937 
7. Rossignol R, Gilkerson R, Aggeler R, Yamagata K, Remington SJ, Capaldi RA. Energy substrate modulates mitochondrial structure and oxidative capacity in cancer cells. Cancer Res (2004) 64(3):985-93. doi:10.1158/0008-5472. CAN-03-1101

8. Mishra P, Chan DC. Metabolic regulation of mitochondrial dynamics. J Cell Biol (2016) 212(4):379-87. doi:10.1083/jcb.201511036

9. Weinberg SE, Sena LA, Chandel NS. Mitochondria in the regulation of innate and adaptive immunity. Immunity (2015) 42(3):406-17. doi:10.1016/j. immuni.2015.02.002

10. Alberts B, Johnson A, Lewis J, Raff M, Roberts K, Walter P. Molecular Biology of the Cell. CT, USA: Taylor \& Francis Inc (2015).

11. Wellen KE, Thompson CB. A two-way street: reciprocal regulation of metabolism and signalling. Nat Rev Mol Cell Biol (2012) 13(4):270-6. doi:10.1038/ nrm3305

12. Takahashi H, McCaffery JM, Irizarry RA, Boeke JD. Nucleocytosolic acetyl-coenzyme a synthetase is required for histone acetylation and global transcription. Mol Cell (2006) 23(2):207-17. doi:10.1016/j.molcel.2006.05.040

13. Westermann B. Molecular machinery of mitochondrial fusion and fission. J Biol Chem (2008) 283(20):13501-5. doi:10.1074/jbc.R800011200

14. Chan DC. Mitochondrial fusion and fission in mammals. Annu Rev Cell Dev Biol (2006) 22:79-99. doi:10.1146/annurev.cellbio.22.010305.104638

15. Rambold AS, Kostelecky B, Elia N, Lippincott-Schwartz J. Tubular network formation protects mitochondria from autophagosomal degradation during nutrient starvation. Proc Natl Acad Sci U S A (2011) 108(25):10190-5. doi:10.1073/pnas.1107402108

16. Youle RJ, van der Bliek AM. Mitochondrial fission, fusion, and stress. Science (2012) 337(6098):1062-5. doi:10.1126/science.1219855

17. Chen H, Chan DC. Mitochondrial dynamics in mammals. Curr Top Dev Biol (2004) 59:119-44. doi:10.1016/S0070-2153(04)59005-1

18. Griparic L, van der Wel NN, Orozco IJ, Peters PJ, van der Bliek AM. Loss of the intermembrane space protein Mgm1/OPAl induces swelling and localized constrictions along the lengths of mitochondria. J Biol Chem (2004) 279(18):18792-8. doi:10.1074/jbc.M400920200

19. Gegg ME, Cooper JM, Chau KY, Rojo M, Schapira AH, Taanman JW. Mitofusin 1 and mitofusin 2 are ubiquitinated in a PINK1/parkin-dependent manner upon induction of mitophagy. Hum Mol Genet (2010) 19(24):4861-70. doi:10.1093/hmg/ddq419

20. Griparic L, Kanazawa T, van der Bliek AM. Regulation of the mitochondrial dynamin-like protein Opal by proteolytic cleavage. J Cell Biol (2007) 178(5):757-64. doi:10.1083/jcb.200704112

21. Ehses S, Raschke I, Mancuso G, Bernacchia A, Geimer S, Tondera D, et al. Regulation of OPA1 processing and mitochondrial fusion by m-AAA protease isoenzymes and OMA1. JCell Biol (2009) 187(7):1023-36. doi:10.1083/ jcb.200906084

22. Zhang K, Li H, Song Z. Membrane depolarization activates the mitochondrial protease OMA1 by stimulating self-cleavage. EMBO Rep (2014) 15(5):576-85. doi:10.1002/embr.201338240

23. Alirol E. Role of hFis1 in Mitochondrial Fission and Apoptosis. Genève: Université de Genève (2006). $117 \mathrm{p}$.

24. James DI, Parone PA, Mattenberger Y, Martinou JC. hFis1, a novel component of the mammalian mitochondrial fission machinery. J Biol Chem (2003) 278(38):36373-9. doi:10.1074/jbc.M303758200

25. Smirnova E, Griparic L, Shurland DL, van der Bliek AM. Dynamin-related protein Drp1 is required for mitochondrial division in mammalian cells. Mol Biol Cell (2001) 12(8):2245-56. doi:10.1091/mbc.12.8.2245

26. Chang CR, Blackstone C. Dynamic regulation of mitochondrial fission through modification of the dynamin-related protein Drpl. Ann N Y Acad Sci (2010) 1201:34-9. doi:10.1111/j.1749-6632.2010.05629.x

27. Leboucher GP, Tsai YC, Yang M, Shaw KC, Zhou M, Veenstra TD, et al. Stress-induced phosphorylation and proteasomal degradation of mitofusin 2 facilitates mitochondrial fragmentation and apoptosis. Mol Cell (2012) 47(4):547-57. doi:10.1016/j.molcel.2012.05.041

28. Buck MD, O'Sullivan D, Klein Geltink RI, Curtis JD, Chang CH, Sanin DE, et al. Mitochondrial dynamics controls T cell fate through metabolic programming. Cell (2016) 166(1):63-76. doi:10.1016/j.cell.2016.05.035

29. Trudeau K, Molina AJA, Roy S. High glucose induces mitochondrial morphology and metabolic changes in retinal pericytes. Invest Ophthalmol Vis Sci (2011) 52(12):8657-64. doi:10.1167/iovs.11-7934
30. Kaech SM, Cui W. Transcriptional control of effector and memory CD8+ $\mathrm{T}$ cell differentiation. Nat Rev Immunol (2012) 12(11):749-61. doi:10.1038/ nri3307

31. Wang R, Dillon CP, Shi LZ, Milasta S, Carter R, Finkelstein D, et al. The transcription factor Myc controls metabolic reprogramming upon $\mathrm{T}$ lymphocyte activation. Immunity (2011) 35(6):871-82. doi:10.1016/j.immuni.2011.09.021

32. Gerriets VA, Rathmell JC. Metabolic pathways in T cell fate and function. Trends Immunol (2012) 33(4):168-73. doi:10.1016/j.it.2012.01.010

33. Ron-Harel N, Santos D, Ghergurovich JM, Sage PT, Reddy A, Lovitch $\mathrm{SB}$, et al. Mitochondrial biogenesis and proteome remodeling promote one-carbon metabolism for T cell activation. Cell Metab (2016) 24(1):104-17. doi:10.1016/j.cmet.2016.06.007

34. Ma EH, Bantug G, Griss T, Condotta S, Johnson RM, Samborska B, et al. Serine is an essential metabolite for effector T cell expansion. Cell Metab (2017) 25(2):345-57. doi:10.1016/j.cmet.2017.01.014

35. van der Windt GJ, Everts B, Chang CH, Curtis JD, Freitas TC, Amiel E, et al. Mitochondrial respiratory capacity is a critical regulator of CD8+ $\mathrm{T}$ cell memory development. Immunity (2012) 36(1):68-78. doi:10.1016/j. immuni.2011.12.007

36. Cui G, Staron MM, Gray SM, Ho PC, Amezquita RA, Wu J, et al. IL-7-induced glycerol transport and TAG synthesis promotes memory CD8+ T cell longevity. Cell (2015) 161(4):750-61. doi:10.1016/j.cell.2015.03.021

37. Michalek RD, Gerriets VA, Jacobs SR, Macintyre AN, MacIver NJ, Mason EF, et al. Cutting edge: distinct glycolytic and lipid oxidative metabolic programs are essential for effector and regulatory CD4+ T cell subsets. J Immunol (2011) 186(6):3299-303. doi:10.4049/jimmunol.1003613

38. Shi LZ, Wang R, Huang G, Vogel P, Neale G, Green DR, et al. HIFlalphadependent glycolytic pathway orchestrates a metabolic checkpoint for the differentiation of TH17 and Treg cells. J Exp Med (2011) 208(7):1367-76. doi:10.1084/jem.20110278

39. Swamy M, Pathak S, Grzes KM, Damerow S, Sinclair LV, van Aalten DM, et al. Glucose and glutamine fuel protein O-GlcNAcylation to control T cell self-renewal and malignancy. Nat Immunol (2016) 17(6):712-20. doi:10.1038/ ni.3439

40. Wei J, Raynor J, Nguyen TM, Chi H. Nutrient and metabolic sensing in T cell responses. Front Immunol (2017) 8:247. doi:10.3389/fimmu.2017.00247

41. van der Windt GJ, O'Sullivan D, Everts B, Huang SC, Buck MD, Curtis JD, et al. CD8 memory $\mathrm{T}$ cells have a bioenergetic advantage that underlies their rapid recall ability. Proc Natl Acad Sci U S A (2013) 110(35):14336-41. doi:10.1073/pnas.1221740110

42. Sukumar M, Liu J, Ji Y, Subramanian M, Crompton JG, Yu Z, et al. Inhibiting glycolytic metabolism enhances CD8+ T cell memory and antitumor function. J Clin Invest (2013) 123(10):4479-88. doi:10.1172/JCI69589

43. Kesarwani P, Murali AK, Al-Khami AA, Mehrotra S. Redox regulation of T-cell function: from molecular mechanisms to significance in human health and disease. Antioxid Redox Signal (2013) 18(12):1497-534. doi:10.1089/ ars. 2011.4073

44. Gubser PM, Bantug GR, Razik L, Fischer M, Dimeloe S, Hoenger G, et al. Rapid effector function of memory CD8+ T cells requires an immediate-early glycolytic switch. Nat Immunol (2013) 14(10):1064-72. doi:10.1038/ni.2687

45. Gomes LC, Di Benedetto G, Scorrano L. During autophagy mitochondria elongate, are spared from degradation and sustain cell viability. Nat Cell Biol (2011) 13(5):589-98. doi:10.1038/ncb2220

46. Cribbs JT, Strack S. Reversible phosphorylation of Drp1 by cyclic AMPdependent protein kinase and calcineurin regulates mitochondrial fission and cell death. EMBO Rep (2007) 8(10):939-44. doi:10.1038/sj.embor.7401062

47. Cereghetti GM, Stangherlin A, Martins de Brito O, Chang CR, Blackstone C, Bernardi $\mathrm{P}$, et al. Dephosphorylation by calcineurin regulates translocation of Drp1 to mitochondria. Proc Natl Acad Sci U S A (2008) 105(41):15803-8. doi:10.1073/pnas.0808249105

48. Baixauli F, Martín-Cófreces NB, Morlino G, Carrasco YR, Calabia-Linares $\mathrm{C}$, Veiga E, et al. The mitochondrial fission factor dynamin-related protein 1 modulates T-cell receptor signalling at the immune synapse. EMBO J (2011) 30(7):1238-50. doi:10.1038/emboj.2011.25

49. Mishra P, Carelli V, Manfredi G, Chan DC. Proteolytic cleavage of Opal stimulates mitochondrial inner membrane fusion and couples fusion to oxidative phosphorylation. Cell Metab (2014) 19(4):630-41. doi:10.1016/j. cmet.2014.03.011 
50. Shutt T, Geoffrion M, Milne R, McBride HM. The intracellular redox state is a core determinant of mitochondrial fusion. EMBO Rep (2012) 13(10):909-15. doi:10.1038/embor.2012.128

51. Samant SA, Zhang HJ, Hong Z, Pillai VB, Sundaresan NR, Wolfgeher D, et al. SIRT3 deacetylates and activates OPA1 to regulate mitochondrial dynamics during stress. Mol Cell Biol (2014) 34(5):807-19. doi:10.1128/ MCB.01483-13

52. Tyrakis PA, Palazon A, Macias D, Lee KL, Phan AT, Veliça P, et al. S-2hydroxyglutarate regulates CD8+ T-lymphocyte fate. Nature (2016) 540(7632):236-41. doi:10.1038/nature20165

53. Carey BW, Finley LW, Cross JR, Allis CD, Thompson CB. Intracellular alpha-ketoglutarate maintains the pluripotency of embryonic stem cells. Nature (2015) 518(7539):413-6. doi:10.1038/nature13981

54. TeSlaa T, Chaikovsky AC, Lipchina I, Escobar SL, Hochedlinger K, Huang $\mathrm{J}$, et al. alpha-Ketoglutarate accelerates the initial differentiation of primed human pluripotent stem cells. Cell Metab (2016) 24(3):485-93. doi:10.1016/j. cmet.2016.07.002

55. Wherry EJ, Kurachi M. Molecular and cellular insights into T cell exhaustion. Nat Rev Immunol (2015) 15(8):486-99. doi:10.1038/nri3862

56. Pauken KE, Wherry EJ. Overcoming T cell exhaustion in infection and cancer. Trends Immunol (2015) 36(4):265-76. doi:10.1016/j.it.2015.02.008

57. Bengsch B, Johnson AL, Kurachi M, Odorizzi PM, Pauken KE, Attanasio J, et al. Bioenergetic insufficiencies due to metabolic alterations regulated by the inhibitory receptor PD-1 are an early driver of CD8(+) T cell exhaustion. Immunity (2016) 45(2):358-73. doi:10.1016/j.immuni.2016.07.008

58. Petrovas C, Mueller YM, Dimitriou ID, Altork SR, Banerjee A, Sklar P, et al. Increased mitochondrial mass characterizes the survival defect of HIV-specific CD8(+) T cells. Blood (2007) 109(6):2505-13. doi:10.1182/ blood-2006-05-021626

59. Ho PC, Bihuniak JD, Macintyre AN, Staron M, Liu X, Amezquita R, et al. Phosphoenolpyruvate is a metabolic checkpoint of anti-tumor T cell responses. Cell (2015) 162(6):1217-28. doi:10.1016/j.cell.2015.08.012

60. Scharping NE, Menk AV, Moreci RS, Whetstone RD, Dadey RE, Watkins SC, et al. The tumor microenvironment represses $\mathrm{T}$ cell mitochondrial biogenesis to drive intratumoral $\mathrm{T}$ cell metabolic insufficiency and dysfunction. Immunity (2016) 45(2):374-88. doi:10.1016/j.immuni.2016.08.009

61. Mockler MB, Conroy MJ, Lysaght J. Targeting T cell immunometabolism for cancer immunotherapy; understanding the impact of the tumor microenvironment. Front Oncol (2014) 4:107. doi:10.3389/fonc.2014.00107

62. Patsoukis N, Bardhan K, Chatterjee P, Sari D, Liu B, Bell LN, et al. PD-1 alters T-cell metabolic reprogramming by inhibiting glycolysis and promoting lipolysis and fatty acid oxidation. Nat Commun (2015) 6:6692. doi:10.1038/ ncomms7692

63. Bratic A, Larsson NG. The role of mitochondria in aging. J Clin Invest (2013) 123(3):951-7. doi:10.1172/JCI64125

64. Harman D. Aging: a theory based on free radical and radiation chemistry. J Gerontol (1956) 11(3):298-300. doi:10.1093/geronj/11.3.298
65. Moiseeva O, Bourdeau V, Roux A, Deschênes-Simard X, Ferbeyre G. Mitochondrial dysfunction contributes to oncogene-induced senescence. $\mathrm{Mol}$ Cell Biol (2009) 29(16):4495-507. doi:10.1128/MCB.01868-08

66. Cui H, Kong Y, Zhang H. Oxidative stress, mitochondrial dysfunction, and aging. J Signal Transduct (2012) 2012:646354. doi:10.1155/2012/646354

67. Salam N, Rane S, Das R, Faulkner M, Gund R, Kandpal U, et al. T cell ageing: effects of age on development, survival \& function. Indian J Med Res (2013) 138(5):595-608.

68. Lin SJ, Guarente L. Nicotinamide adenine dinucleotide, a metabolic regulator of transcription, longevity and disease. Curr Opin Cell Biol (2003) 15(2):241-6. doi:10.1016/S0955-0674(03)00006-1

69. Cohen HY, Miller C, Bitterman KJ, Wall NR, Hekking B, Kessler B, et al. Calorie restriction promotes mammalian cell survival by inducing the SIRT1 deacetylase. Science (2004) 305(5682):390-2. doi:10.1126/science.1099196

70. Anderson RM, Barger JL, Edwards MG, Braun KH, O’Connor CE, Prolla TA, et al. Dynamic regulation of PGC-1alpha localization and turnover implicates mitochondrial adaptation in calorie restriction and the stress response. Aging Cell (2008) 7(1):101-11. doi:10.1111/j.1474-9726.2007.00357.x

71. Pathak S, Mohan C. Cellular and molecular pathogenesis of systemic lupus erythematosus: lessons from animal models. Arthritis Res Ther (2011) 13(5):241. doi:10.1186/ar3465

72. Gergely P Jr, Niland B, Gonchoroff N, Pullmann R Jr, Phillips PE, Perl A. Persistent mitochondrial hyperpolarization, increased reactive oxygen intermediate production, and cytoplasmic alkalinization characterize altered IL-10 signaling in patients with systemic lupus erythematosus. J Immunol (2002) 169(2):1092-101. doi:10.4049/jimmunol.169.2.1092

73. Lood C, Blanco LP, Purmalek MM, Carmona-Rivera C, De Ravin SS, Smith CK, et al. Neutrophil extracellular traps enriched in oxidized mitochondrial DNA are interferogenic and contribute to lupus-like disease. Nat Med (2016) 22(2):146-53. doi:10.1038/nm.4027

74. Gergely P Jr, Grossman C, Niland B, Puskas F, Neupane H, Allam F, et al. Mitochondrial hyperpolarization and ATP depletion in patients with systemic lupus erythematosus. Arthritis Rheum (2002) 46(1):175-90. doi:10.1002/1529-0131(200201)46:1<175::AID-ART10015>3.0.CO;2-H

Conflict of Interest Statement: The authors declare no potential conflict of interest with any commercial or financial relationship on this work.

The reviewer, MG, and handling editor declared their shared affiliation, and the handling editor states that the process nevertheless met the standards of a fair and objective review.

Copyright (c) 2017 Chao, Wang and Ho. This is an open-access article distributed under the terms of the Creative Commons Attribution License (CC BY). The use, distribution or reproduction in other forums is permitted, provided the original author(s) or licensor are credited and that the original publication in this journal is cited, in accordance with accepted academic practice. No use, distribution or reproduction is permitted which does not comply with these terms. 